\title{
A New Family of $q$-Supercongruences Modulo the Fourth Power of a Cyclotomic Polynomial
}

\author{
Victor J. W. Guo and Michael J. Schlosser@
}

\begin{abstract}
We establish a new family of $q$-supercongruences modulo the fourth power of a cyclotomic polynomial, and give several related results. Our main ingredients are $q$-microscoping and the Chinese remainder theorem for polynomials.
\end{abstract}

Mathematics Subject Classification. Primary 33D15, Secondary 11A07, $11 \mathrm{~B} 65$.

Keywords. Basic hypergeometric series, supercongruences, $q$-congruences, cyclotomic polynomial, $q$-microscoping, Chinese remainder theorem for polynomials.

\section{Introduction}

More than one hundred years ago, Ramanujan mysteriously recorded a list of rapidly convergent series of $1 / \pi$ (see $[1$, p. 352]), including

$$
\sum_{n=0}^{\infty} \frac{\left(\begin{array}{c}
4 n \\
2 n
\end{array}\right)\left(\begin{array}{c}
2 n \\
n
\end{array}\right)^{2}}{2^{8 n} 3^{2 n}}(8 n+1)=\frac{2 \sqrt{3}}{\pi},
$$

which he later published in [19, Equation (40)]. In 1997, Van Hamme [23] observed that 13 Ramanujan's and Ramanujan-type formulas possess interesting $p$-adic analogues, such as

Victor J. W. Guo was partially supported by the National Natural Science Foundation of China (Grant 11771175). Michael J. Schlosser was partially supported by FWF Austrian Science Fund Grant P32305. 


$$
\sum_{k=0}^{(p-1) / 2}(4 k+1) \frac{\left(\frac{1}{2}\right)_{k}^{4}}{k !^{4}} \equiv p \quad\left(\bmod p^{3}\right)
$$

where $p>3$ is a prime and $(a)_{n}=a(a+1) \cdots(a+n-1)$ is the Pochhammer symbol. Van Hamme [23, (C.2)] himself proved (1.2) and two of the other supercongruences of his list. The supercongruence (1.2) was later proved to be true modulo $p^{4}$ by Long [17]. For more Ramanujan-type supercongruences, we refer the reader to Zudilin's paper [26].

During the past few years, $q$-analogues of congruences and supercongruences have been investigated by many authors (see [3-16, 18, 21,22, 24, 25, 27]). For instance, using a method similar to that used in [26], the first author and Wang [12, Theorem 1.2] gave a $q$-analogue of (1.2): for odd $n$,

$$
\begin{aligned}
& \sum_{k=0}^{(n-1) / 2}[4 k+1] \frac{\left(q ; q^{2}\right)_{k}^{4}}{\left(q^{2} ; q^{2}\right)_{k}^{4}} \\
& \quad \equiv q^{(1-n) / 2}[n]+\frac{\left(n^{2}-1\right)(1-q)^{2}}{24} q^{(1-n) / 2}[n]^{3} \quad\left(\bmod [n] \Phi_{n}(q)^{3}\right) .
\end{aligned}
$$

Moreover, the first author and Zudilin [13] devised a method of 'creative microscoping' to prove that, for any positive integer $n$ with $\operatorname{gcd}(n, 6)=1$,

$$
\begin{aligned}
& \sum_{k=0}^{(n-1) / 2}[8 k+1] \frac{\left(q ; q^{2}\right)_{k}^{2}\left(q ; q^{2}\right)_{2 k}}{\left(q^{2} ; q^{2}\right)_{2 k}\left(q^{6} ; q^{6}\right)_{k}^{2}} q^{2 k^{2}} \\
& \equiv q^{-(n-1) / 2}[n]\left(\frac{-3}{n}\right) \quad\left(\bmod [n] \Phi_{n}(q)^{2}\right)
\end{aligned}
$$

where $\left(\frac{-3}{-}\right)$ is the Jacobi symbol, see [13, Theorem 1.1, Equation (6)]. Here it is appropriate to recall the standard $q$-hypergeometric notation: $(a ; q)_{n}=(1-$ $a)(1-a q) \cdots\left(1-a q^{n-1}\right)$ is the $q$-shifted factorial, with the condensed notation $\left(a_{1}, \ldots, a_{m} ; q\right)_{n}=\left(a_{1} ; q\right)_{n} \cdots\left(a_{m} ; q\right)_{n}$ for products of $q$-shifted factorials; $[n]=$ $[n]_{q}=\left(1-q^{n}\right) /(1-q)$ is the $q$-integer; and $\Phi_{n}(q)$ stands for the $n$th cyclotomic polynomial in $q$ :

$$
\Phi_{n}(q)=\prod_{\substack{1 \leqslant k \leqslant n \\ \operatorname{gcd}(k, n)=1}}\left(q-\zeta_{n}^{k}\right)
$$

where $\zeta_{n}$ denotes an $n$th primitive root of unity.

Clearly, the $q$-supercongruence (1.4) is a $q$-analogue of the following result (see [20, Conjecture 5.6]):

$$
\sum_{k=0}^{(p-1) / 2}(8 k+1) \frac{\left(\begin{array}{c}
4 k \\
2 k
\end{array}\right)\left(\begin{array}{c}
2 k \\
k
\end{array}\right)^{2}}{2^{8 k} 3^{2 k}} \equiv p\left(\frac{-3}{p}\right) \quad\left(\bmod p^{3}\right) \quad \text { for } p>3 \text { prime }
$$


which is a $p$-adic analogue of (1.1). This means that by letting $q \rightarrow 1$ in (1.4) one obtains (1.5). We point out that no other proofs of (1.5) are known up to now.

The first author and Zudilin [13, Theorem 4.2] also gave a two-parameter generalization of (1.3) as follows: for odd $n$, modulo $[n]\left(1-a q^{n}\right)\left(a-q^{n}\right)$,

$$
\sum_{k=0}^{m}[4 k+1] \frac{\left(a q, q / a, q / b, q ; q^{2}\right)_{k}}{\left(a q^{2}, q^{2} / a, b q^{2}, q^{2} ; q^{2}\right)_{k}} b^{k} \equiv \frac{(b / q)^{(n-1) / 2}\left(q^{2} / b ; q^{2}\right)_{(n-1) / 2}}{\left(b q^{2} ; q^{2}\right)_{(n-1) / 2}}[n],
$$

where $m=(n-1) / 2$ or $(n-1)$. Recently, based on the above $q$-congruence, by applying the Chinese remainder theorem for coprime polynomials, the first author [6, Theorem 1.1] succeeded in giving a full parametric generalization of (1.3): modulo $[n] \Phi_{n}(q)\left(1-a q^{n}\right)\left(a-q^{n}\right)$,

$$
\begin{aligned}
& \sum_{k=0}^{m}[4 k+1] \frac{\left(a q, q / a, q, q ; q^{2}\right)_{k}}{\left(a q^{2}, q^{2} / a, q^{2}, q^{2} ; q^{2}\right)_{k}} \\
& \quad \equiv q^{(1-n) / 2}[n]+q^{(1-n) / 2}[n] \frac{\left(1-a q^{n}\right)\left(a-q^{n}\right)}{(1-a)^{2}}\left(1-\frac{n(1-a) a^{(n-1) / 2}}{1-a^{n}}\right),
\end{aligned}
$$

where $m=(n-1) / 2$ or $(n-1)$. Moreover, the present authors [8, Theorem 1.1, Equation (2a)] showed that, for odd $n>1$,

$$
\sum_{k=0}^{(n+1) / 2}[4 k-1] \frac{\left(q^{-1} ; q^{2}\right)_{k}^{4}}{\left(q^{2} ; q^{2}\right)_{k}^{4}} q^{4 k} \equiv-\left(1+3 q+q^{2}\right)[n]^{4} \quad\left(\bmod [n]^{4} \Phi_{n}(q)\right) .
$$

The main purpose of this paper is to establish a new family of $q$ supercongruences modulo the fourth power of a cyclotomic polynomial, which may somewhat be deemed a generalization of (1.3) and (1.7) modulo $[n] \Phi_{n}(q)^{3}$.

Theorem 1.1. Let $d, n, r$ be integers satisfying $d \geqslant 2, r \leqslant d-2$ (in particular, $r$ may be negative), and $n \geqslant d-r$, such that $d$ and $r$ are coprime, and $n \equiv-r$ $(\bmod d)$. Then

$$
\begin{aligned}
& \sum_{k=0}^{M}[2 d k+r] \frac{\left(q^{r} ; q^{d}\right)_{k}^{4}}{\left(q^{d} ; q^{d}\right)_{k}^{4}} q^{(d-2 r) k}
\end{aligned}
$$

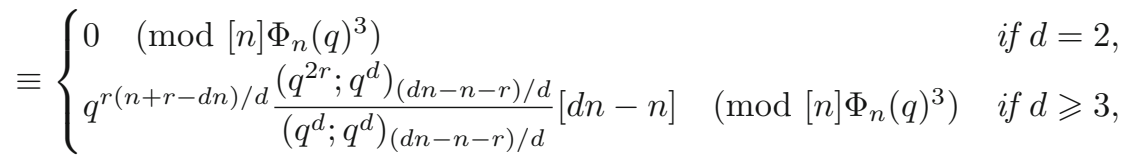

where $M=(d n-n-r) / d$ or $n-1$.

The proof is given in Sect. 3 .

It is easy to see that the $q$-factorial $\left(q^{2 r} ; q^{d}\right)_{(d n-n-r) / d}$ in (1.8) contains the factor $1-q^{(d-2) n}$ for $d \geqslant 3$. Since $1-q^{(d-2) n} \equiv[d n-n] \equiv 0\left(\bmod \Phi_{n}(q)\right)$ 
and $\left(q^{d} ; q^{d}\right)_{(d n-n-r) / d}$ is coprime with $\Phi_{n}(q)$, we conclude from Theorem 1.1 that

$$
\sum_{k=0}^{n-1}[2 d k+r] \frac{\left(q^{r} ; q^{d}\right)_{k}^{4}}{\left(q^{d} ; q^{d}\right)_{k}^{4}} q^{(d-2 r) k} \equiv 0 \quad\left(\bmod \Phi_{n}(q)^{2}\right)
$$

for $d \geqslant 3$. Our proof of Theorem 1.1 implies that the above $q$-congruence is further true modulo $[n] \Phi_{n}(q)$. Or the reader may check that the denominator of the reduced form of the fraction $\left(q^{2 r} ; q^{d}\right)_{(d n-n-r) / d} /\left(q^{d} ; q^{d}\right)_{(d n-n-r) / d}$ is coprime with $[n]$.

We should point out that the present authors [10] have given a different generalization of (1.3) and (1.7) modulo $\Phi_{n}(q)^{4}$ as follows: for $d \geqslant 3$ and $n, r$ satisfying the same condition as in Theorem 1.1, we have

$$
\sum_{k=0}^{n-1}[2 d k+r] \frac{\left(q^{r} ; q^{d}\right)_{k}^{2 d}}{\left(q^{d} ; q^{d}\right)_{k}^{2 d}} q^{d(d-1-r) k} \equiv 0 \quad\left(\bmod \Phi_{n}(q)^{4}\right) .
$$

For $d \geqslant 3$ and $r= \pm 1$, Theorem 1.1 can be stated as follows:

Corollary 1.2. Let $d$ and $n$ be positive integers with $d \geqslant 3$ and $n \equiv-1$ $(\bmod d)$. Then

$$
\begin{aligned}
& \sum_{k=0}^{M}[2 d k+1] \frac{\left(q ; q^{d}\right)_{k}^{4}}{\left(q^{d} ; q^{d}\right)_{k}^{4}} q^{(d-2) k} \\
& \quad \equiv q^{(n-d n+1) / d} \frac{\left(q^{2} ; q^{d}\right)_{(d n-n-1) / d}}{\left(q^{d} ; q^{d}\right)_{(d n-n-1) / d}}[d n-n] \quad\left(\bmod [n] \Phi_{n}(q)^{3}\right),
\end{aligned}
$$

where $M=(d n-n-1) / d$ or $n-1$.

Corollary 1.3. Let $d$ and $n$ be positive integers with $d \geqslant 3$ and $n \equiv 1(\bmod d)$. Then

$$
\begin{aligned}
& \sum_{k=0}^{M}[2 d k-1] \frac{\left(q^{-1} ; q^{d}\right)_{k}^{4}}{\left(q^{d} ; q^{d}\right)_{k}^{4}} q^{(d+2) k} \\
& \quad \equiv q^{(d n-n+1) / d} \frac{\left(q^{-2} ; q^{d}\right)_{(d n-n+1) / d}}{\left(q^{d} ; q^{d}\right)_{(d n-n+1) / d}}[d n-n] \quad\left(\bmod [n] \Phi_{n}(q)^{3}\right),
\end{aligned}
$$

where $M=(d n-n+1) / d$ or $n-1$.

We shall also prove the following $q$-congruence, which was originally conjectured by the first author and Zudilin [13, Conjecture 5.2].

Theorem 1.4. Let $d$ and $n$ be positive integers with $d \geqslant 3$ and $n \equiv-1(\bmod d)$. Then

$$
\sum_{k=0}^{n-1}[2 d k+1] \frac{\left(a q, q / a ; q^{d}\right)_{k}\left(q ; q^{d}\right)_{k}^{2}}{\left(a q^{d}, q^{d} / a ; q^{d}\right)_{k}\left(q^{d} ; q^{d}\right)_{k}^{2}} q^{(d-2) k} \equiv 0 \quad\left(\bmod [n] \Phi_{n}(q)\right) .
$$

Note that the $a=1$ case of (1.10) modulo $\Phi_{n}(q)^{2}$ has already been proved by the present authors [11, Theorem 2.4]. 


\section{Some Lemmas}

We first give the following result which is a generalization of [11, Lemma 3.1], [10, Lemma 3] and [24, (2.1)].

Lemma 2.1. Let $d, m$ and $n$ be positive integers with $m \leqslant n-1$. Let $r$ be an integer satisfying $d m \equiv-r(\bmod n)$. Then, for $0 \leqslant k \leqslant m$, we have

$$
\frac{\left(a q^{r} ; q^{d}\right)_{m-k}}{\left(q^{d} / a ; q^{d}\right)_{m-k}} \equiv(-a)^{m-2 k} \frac{\left(a q^{r} ; q^{d}\right)_{k}}{\left(q^{d} / a ; q^{d}\right)_{k}} q^{m(d m-d+2 r) / 2+(d-r) k} \quad\left(\bmod \Phi_{n}(q)\right) .
$$

Proof. In view of $q^{d m+r} \equiv q^{n} \equiv 1\left(\bmod \Phi_{n}(q)\right)$, we have

$$
\begin{aligned}
\frac{\left(a q^{r} ; q^{d}\right)_{m}}{\left(q^{d} / a ; q^{d}\right)_{m}} & =\frac{\left(1-a q^{r}\right)\left(1-a q^{d+r}\right) \cdots\left(1-a q^{d m-d+r}\right)}{\left(1-q^{d} / a\right)\left(1-q^{2 d} / a\right) \cdots\left(1-q^{d m} / a\right)} \\
& \equiv \frac{\left(1-a q^{r}\right)\left(1-a q^{d+r}\right) \cdots\left(1-a q^{d m-d+r}\right)}{\left(1-q^{d-d m-r} / a\right)\left(1-q^{2 d-d m-r} / a\right) \cdots\left(1-q^{-r} / a\right)} \\
& =(-a)^{m} q^{m(d m-d+2 r) / 2}\left(\bmod \Phi_{n}(q)\right) .
\end{aligned}
$$

Furthermore, modulo $\Phi_{n}(q)$, we get

$$
\begin{aligned}
& \frac{\left(a q^{r} ; q^{d}\right)_{m-k}}{\left(q^{d} / a ; q^{d}\right)_{m-k}} \\
& \quad=\frac{\left(a q^{r} ; q^{d}\right)_{m}}{\left(q^{d} / a ; q^{d}\right)_{m}} \frac{\left(1-q^{d m-d k+d} / a\right)\left(1-q^{d m-d k+2 d} / a\right) \cdots\left(1-q^{d m} / a\right)}{\left(1-a q^{d m-d k+r}\right)\left(1-a q^{d m-d k+d+r}\right) \cdots\left(1-a q^{d m-d+r}\right)} \\
& \quad \equiv \frac{\left(a q^{r} ; q^{d}\right)_{m}}{\left(q^{d} / a ; q^{d}\right)_{m}} \frac{\left(1-q^{d-d k-r} / a\right)\left(1-q^{2 d-d k-r} / a\right) \cdots\left(1-q^{-r} / a\right)}{\left(1-a q^{-d k}\right)\left(1-a q^{d-d k}\right) \cdots\left(1-a q^{-d}\right)} \\
& \quad=\frac{\left(a q^{r} ; q^{d}\right)_{m}}{\left(q^{d} / a ; q^{d}\right)_{m}} \frac{\left(a q^{r} ; q^{d}\right)_{k}}{\left(q^{d} / a ; q^{d}\right)_{k}} a^{-2 k} q^{(d-r) k},
\end{aligned}
$$

which together with (2.1) establishes the assertion.

Lemma 2.2. Let $d, n$ be positive integers with $\operatorname{gcd}(d, n)=1$. Let $r$ be an integer and let $a, b$ be indeterminates. Then

$$
\begin{aligned}
& \sum_{k=0}^{m}[2 d k+r] \frac{\left(a q^{r}, q^{r} / a, q^{r} / b, q^{r} ; q^{d}\right)_{k}}{\left(a q^{d}, q^{d} / a, b q^{d}, q^{d} ; q^{d}\right)_{k}} b^{k} q^{(d-2 r) k} \equiv 0 \quad(\bmod [n]), \\
& \sum_{k=0}^{n-1}[2 d k+r] \frac{\left(a q^{r}, q^{r} / a, q^{r} / b, q^{r} ; q^{d}\right)_{k}}{\left(a q^{d}, q^{d} / a, b q^{d}, q^{d} ; q^{d}\right)_{k}} b^{k} q^{(d-2 r) k} \equiv 0 \quad(\bmod [n]),
\end{aligned}
$$

where $0 \leqslant m \leqslant n-1$ and $d m \equiv-r(\bmod n)$.

Proof. It is clear that Lemma 2.2 is true for $n=1$ or $r=0$. We now assume that $n>1$ and $r \neq 0$. By Lemma 2.1 (which is clearly also true for $m=0$ ) 
one sees that, for $0 \leqslant k \leqslant m$, the $k$ th and $(m-k)$ th terms on the left-hand side of (2.2) cancel each other modulo $\Phi_{n}(q)$, i.e.,

$$
\begin{aligned}
& {[2 d(m-k)+r] \frac{\left(a q^{r}, q^{r} / a, q^{r} / b, q^{r} ; q^{d}\right)_{m-k}}{\left(a q^{d}, q^{d} / a, b q^{d}, q^{d} ; q^{d}\right)_{m-k}} b^{m-k} q^{(d-2 r)(m-k)}} \\
& \quad \equiv-[2 d k+r] \frac{\left(a q^{r}, q^{r} / a, q^{r} / b, q^{r} ; q^{d}\right)_{k}}{\left(a q^{d}, q^{d} / a, b q^{d}, q^{d} ; q^{d}\right)_{k}} b^{k} q^{(d-2 r) k} \quad\left(\bmod \Phi_{n}(q)\right) .
\end{aligned}
$$

This proves that the $q$-congruence $(2.2)$ holds modulo $\Phi_{n}(q)$.

Moreover, since $d m \equiv-r(\bmod n)$, the expression $\left(q^{r} ; q^{d}\right)_{k}$ contains a factor of the form $1-q^{\alpha n}$ for $m<k \leqslant n-1$, and is therefore congruent to 0 modulo $\Phi_{n}(q)$. At the same time the expression $\left(q^{d} ; q^{d}\right)_{k}$ is relatively prime to $\Phi_{n}(q)$ for $m<k \leqslant n-1$. Therefore, each summand in (2.3) with $k$ in the range $m<k \leqslant n-1$ is congruent to 0 modulo $\Phi_{n}(q)$. This together with (2.2) modulo $\Phi_{n}(q)$ establishes the $q$-congruence $(2.3)$ modulo $\Phi_{n}(q)$.

We are now able to prove (2.2) and (2.3) modulo $[n]$. Let $\zeta \neq 1$ be an $n$th root of unity, not necessarily primitive. Namely, $\zeta$ is a primitive root of unity of degree $s$ with $s \mid n$ and $s>1$. Let $c_{q}(k)$ denote the $k$ th term on the left-hand side of (2.3), i.e.,

$$
c_{q}(k)=[2 d k+r] \frac{\left(a q^{r}, q^{r} / a, q^{r} / b, q^{r} ; q^{d}\right)_{k}}{\left(a q^{d}, q^{d} / a, b q^{d}, q^{d} ; q^{d}\right)_{k}} b^{k} q^{(d-2 r) k} .
$$

The $q$-congruences (2.2) and (2.3) modulo $\Phi_{n}(q)$ with $n \mapsto s$ imply that

$$
\sum_{k=0}^{m_{1}} c_{\zeta}(k)=\sum_{k=0}^{s-1} c_{\zeta}(k)=0,
$$

where $d m_{1} \equiv-r(\bmod s)$ and $0 \leqslant m_{1} \leqslant s-1$. We have

$$
\lim _{q \rightarrow \zeta} \frac{c_{q}(\ell s+k)}{c_{q}(\ell s)}=\frac{c_{\zeta}(\ell s+k)}{c_{\zeta}(\ell s)}=\frac{c_{\zeta}(k)}{[r]} .
$$

It follows that

$$
\sum_{k=0}^{n-1} c_{\zeta}(k)=\sum_{\ell=0}^{n / s-1} \sum_{k=0}^{s-1} c_{\zeta}(\ell s+k)=\frac{1}{[r]} \sum_{\ell=0}^{n / s-1} c_{\zeta}(\ell s) \sum_{k=0}^{s-1} c_{\zeta}(k)=0,
$$

and

$$
\sum_{k=0}^{m} c_{\zeta}(k)=\frac{1}{[r]} \sum_{\ell=0}^{\left(m-m_{1}\right) / s-1} c_{\zeta}(\ell s) \sum_{k=0}^{s-1} c_{\zeta}(k)+\frac{c_{\zeta}\left(m-m_{1}\right)}{[r]} \sum_{k=0}^{m_{1}} c_{\zeta}(k)=0 .
$$

This means that the sums $\sum_{k=0}^{n-1} c_{q}(k)$ and $\sum_{k=0}^{m} c_{q}(k)$ are both divisible by the cyclotomic polynomial $\Phi_{s}(q)$. Since this is true for any divisor $s>1$ of $n$, we deduce that they are divisible by 


$$
\prod_{s \mid n, s>1} \Phi_{s}(q)=[n]
$$

thus establishing the $q$-congruences (2.2) and (2.3).

We now give the following result, which is a generalization of $[13$, Theorem $4.2]$.

Lemma 2.3. Let $d, n, r$ be integers satisfying $d \geqslant 2$ and $n \geqslant d-r$, such that $d$ and $r$ are coprime, and $n \equiv-r(\bmod d)$. Let $a, b$ be indeterminates. Then, modulo $[n]\left(1-a q^{d n-n}\right)\left(a-q^{d n-n}\right)$,

$$
\begin{aligned}
& \sum_{k=0}^{M}[2 d k+r] \frac{\left(a q^{r}, q^{r} / a, q^{r} / b, q^{r} ; q^{d}\right)_{k}}{\left(a q^{d}, q^{d} / a, b q^{d}, q^{d} ; q^{d}\right)_{k}} b^{k} q^{(d-2 r) k} \\
& \quad \equiv \frac{\left(q^{2 r} / b ; q^{d}\right)_{(d n-n-r) / d}}{\left(b q^{d} ; q^{d}\right)_{(d n-n-r) / d}}[d n-n]\left(\frac{b}{q^{r}}\right)^{(d n-n-r) / d}
\end{aligned}
$$

where $M=(d n-n-r) / d$ or $n-1$.

Proof. By the condition in the lemma, we have $r \neq 0$. Recall that Jackson's ${ }_{6} \phi_{5}$ summation formula can be written as

$$
\sum_{k=0}^{N} \frac{\left(1-a q^{2 k}\right)\left(a, b, c, q^{-N} ; q\right)_{k}}{(1-a)\left(q, a q / b, a q / c, a q^{N+1} ; q\right)_{k}}\left(\frac{a q^{N+1}}{b c}\right)^{k}=\frac{(a q, a q / b c ; q)_{N}}{(a q / b, a q / c ; q)_{N}}
$$

(see [2, Appendix (II.21)]). Letting $q \mapsto q^{d}, a=q^{r}, b \mapsto q^{r} / b, c=q^{d n-n+r}$ and $N=(d n-n-r) / d$ in $(2.7)$, we obtain

$$
\begin{aligned}
& \sum_{k=0}^{M} \frac{[2 d k+r]\left(q^{r-d n+n}, q^{r+d n-n}, q^{r} / b, q^{r} ; q^{d}\right)_{k}}{[r]\left(q^{d-d n+n}, q^{d+d n-n}, b q^{d}, q^{d} ; q^{d}\right)_{k}} q^{(d-2 r) k} \\
& \quad=\frac{\left(q^{d+r}, b q^{d-d n+n-r} ; q^{d}\right)_{(d n-n-r) / d}}{\left(b q^{d}, q^{d-d n+n} ; q^{d}\right)_{(d n-n-r) / d}} \\
& \quad=\frac{\left(q^{2 r} / b ; q^{d}\right)_{(d n-n-r) / d}[d n-n]}{\left(b q^{d} ; q^{d}\right)_{(d n-n-r) / d}[r]}\left(\frac{b}{q^{r}}\right)^{(d n-n-r) / d} .
\end{aligned}
$$

Namely, when $a=q^{d n-n}$ or $a=q^{n-d n}$ the two sides of (2.6) are equal. Thus, the $q$-congruence $(2.6)$ holds modulo $\left(1-a q^{d n-n}\right)\left(a-q^{d n-n}\right)$.

On the other hand, by Lemma 2.2, we see that the left-hand side of (2.6) is congruent to 0 modulo $[n]$. Since $[d n-n]$ is also congruent to 0 modulo $[n]$ and $\left(b q^{d} ; q^{d}\right)_{(d n-n-r) / d}$ is coprime with $[n]$, we conclude that (2.6) also holds modulo $[n]$. The proof then follows from the fact that $\left(1-a q^{d n-n}\right)\left(a-q^{d n-n}\right)$ and $[n]$ are coprime polynomials.

Note that the condition $n \geqslant d-r$ means that $(d n-n-r) / d \leqslant n-1$, which is used in the identity (2.8) for $M=n-1$. 
Lemma 2.4. Let $d, n, r$ be integers satisfying $d \geqslant 2$ and $n \geqslant d-r$, such that $d$ and $r$ are coprime, and $n \equiv-r(\bmod d)$. Let $a, b$ be indeterminates. Then

$$
\begin{aligned}
& \sum_{k=0}^{M}[2 d k+r] \frac{\left(a q^{r}, q^{r} / a, q^{r} / b, q^{r} ; q^{d}\right)_{k}}{\left(a q^{d}, q^{d} / a, b q^{d}, q^{d} ; q^{d}\right)_{k}} b^{k} q^{(d-2 r) k} \\
& \quad \equiv \frac{\left(q^{r}, q^{d-r} ; q^{d}\right)_{(d n-n-r) / d}}{\left(a q^{d}, q^{d} / a ; q^{d}\right)_{(d n-n-r) / d}}[d n-n] \quad\left(\bmod b-q^{d n-n}\right),
\end{aligned}
$$

where $M=(d n-n-r) / d$ or $n-1$.

Proof. Letting $q \mapsto q^{d}$ and taking $a=q^{r}, b=a q^{r}, c=q^{r} / a$ and $N=$ $(d n-n-r) / d$ in $(2.7)$, we obtain

$$
\begin{aligned}
& \sum_{k=0}^{(d n-n-r) / d} \frac{[2 d k+r]\left(a q^{r}, q^{r} / a, q^{r-d n+n}, q^{r} ; q^{d}\right)_{k}}{[r]\left(a q^{d}, q^{d} / a, q^{d+d n-n}, q^{d} ; q^{d}\right)_{k}} q^{(d n-n+d-2 r) k} \\
& \quad=\frac{\left(q^{d+r}, q^{d-r} ; q^{d}\right)_{(d n-n-r) / d}}{\left(a q^{d}, q^{d} / a ; q^{d}\right)_{(d n-n-r) / d}} .
\end{aligned}
$$

Namely, when $b=q^{d n-n}$ both sides of (2.9) are equal. This proves the desired $q$-congruence.

\section{Proof of Theorems 1.1 and 1.4}

With the help of Lemmas 2.3 and 2.4, we can prove the main theorems in this paper now. We need to establish the following parametric generalization of Theorem 1.1.

Theorem 3.1. Let $d, n, r$ be integers satisfying $d \geqslant 2, r \leqslant d-2$, and $n \geqslant$ $d-r$, such that $d$ and $r$ are coprime, and $n \equiv-r(\bmod d)$. Let $a$ be an indeterminate. Then, modulo $[n] \Phi_{n}(q)\left(1-a q^{d n-n}\right)\left(a-q^{d n-n}\right)$,

$$
\begin{aligned}
& \sum_{k=0}^{M}[2 d k+r] \frac{\left(a q^{r}, q^{r} / a, q^{r}, q^{r} ; q^{d}\right)_{k}}{\left(a q^{d}, q^{d} / a, q^{d}, q^{d} ; q^{d}\right)_{k}} q^{(d-2 r) k} \\
& \equiv \frac{\left(q^{2 r} ; q^{d}\right)_{(d n-n-r) / d}}{\left(q^{d} ; q^{d}\right)_{(d n-n-r) / d}}[d n-n] q^{r(n-d n+r) / d},
\end{aligned}
$$

where $M=(d n-n-r) / d$ or $n-1$.

Proof. It is clear that the polynomials $[n]\left(1-a q^{d n-n}\right)\left(a-q^{d n-n}\right)$ and $b-q^{d n-n}$ are coprime. By the Chinese remainder theorem for coprime polynomials, we can determine the remainder of the left-hand side of (2.6) modulo $[n](1-$ $\left.a q^{d n-n}\right)\left(a-q^{d n-n}\right)\left(b-q^{d n-n}\right)$ from (2.6) and (2.9). To this end, we require the following $q$-congruences: 


$$
\begin{aligned}
\frac{\left(b-q^{d n-n}\right)\left(a b-1-a^{2}+a q^{d n-n}\right)}{(a-b)(1-a b)} & \equiv 1 \quad\left(\bmod \left(1-a q^{d n-n}\right)\left(a-q^{d n-n}\right)\right), \\
\frac{\left(1-a q^{d n-n}\right)\left(a-q^{d n-n}\right)}{(a-b)(1-a b)} & \equiv 1 \quad\left(\bmod b-q^{d n-n}\right) .
\end{aligned}
$$

Thus, from (2.6) and (2.9) we deduce that, modulo $[n]\left(1-a q^{d n-n}\right)\left(a-q^{d n-n}\right)$ $\left(b-q^{d n-n}\right)$,

$$
\begin{aligned}
& \sum_{k=0}^{M}[2 d k+r] \frac{\left(a q^{r}, q^{r} / a, q^{r} / b, q^{r} ; q^{d}\right)_{k}}{\left(a q^{d}, q^{d} / a, b q^{d}, q^{d} ; q^{d}\right)_{k}} b^{k} q^{(d-2 r) k} \\
& \equiv \frac{\left(q^{2 r} / b ; q^{d}\right)_{(d n-n-r) / d}}{\left(b q^{d} ; q^{d}\right)_{(d n-n-r) / d}} \frac{\left(b-q^{d n-n}\right)\left(a b-1-a^{2}+a q^{d n-n}\right)}{(a-b)(1-a b)} \\
& \quad \times[d n-n]\left(b q^{-r}\right)(d n-n-r) / d \\
& \quad+\frac{\left(q^{r}, q^{d-r} ; q^{d}\right)_{(d n-n-r) / d}}{\left(a q^{d}, q^{d} / a ; q^{d}\right)_{(d n-n-r) / d}} \frac{\left(1-a q^{d n-n}\right)\left(a-q^{d n-n}\right)}{(a-b)(1-a b)}[d n-n] .
\end{aligned}
$$

Note that $1-q^{d n-n}$ has the factor $\Phi_{n}(q)$ and so do $\left(q^{2 r} ; q^{d}\right)_{(d n-n-r) / d}$ and $\left(q^{d-r} ; q^{d}\right)_{(d n-n-r) / d}$ since they contain the factors $1-q^{(d-2) n}$ and $1-q^{n}$, respectively (we need to check that $2 r+2 n \leqslant d n$, which is guaranteed by the condition $r \leqslant d-2$ and $n \geqslant d-r$ in the theorem). Moreover, the factor $\left(b q^{d} ; q^{d}\right)_{M}$ in the denominator of the left-hand side of (3.2) is coprime with $\Phi_{n}(q)$ when $b=1$. Thus, letting $b=1$ in (3.2) and observing that

$$
\left(1-q^{d n-n}\right)\left(1+a^{2}-a-a q^{d n-n}\right)=(1-a)^{2}+\left(1-a q^{d n-n}\right)\left(a-q^{d n-n}\right),
$$

we see that the right-hand of (3.2) reduces to

$$
\frac{\left(q^{2 r} ; q^{d}\right)_{(d n-n-r) / d}}{\left(q^{d} ; q^{d}\right)_{(d n-n-r) / d}}[d n-n] q^{r(n-d n+r) / d}
$$

modulo $\Phi_{n}(q)^{2}\left(1-a q^{d n-n}\right)\left(a-q^{d n-n}\right)$, thus establishing Eq. (3.1) modulo $\Phi_{n}(q)^{2}\left(1-a q^{d n-n}\right)\left(a-q^{d n-n}\right)$. On the other hand, the $q$-congruences (2.2) $(m=(d n-n-r) / d$ in this case) and (2.3) are also true for $b=1$. That is, the $q$-congruence (3.1) holds modulo $[n]$. The proof then follows from the fact that the least common multiple of $\Phi_{n}(q)^{2}\left(1-a q^{d n-n}\right)\left(a-q^{d n-n}\right)$ and $[n]$ is just $[n] \Phi_{n}(q)\left(1-a q^{d n-n}\right)\left(a-q^{d n-n}\right)$.

Proof of Theorem 1.1. Since $\left(1-q^{d n-n}\right)^{2}$ contains the factor $\Phi_{n}(q)^{2}$ and $\left(q^{d} ; q^{d}\right)_{M}$ is coprime with $\Phi_{n}(q)$, letting $a=1$ in (3.1), we are led to

$$
\begin{aligned}
& \sum_{k=0}^{M}[2 d k+r] \frac{\left(q^{r} ; q^{d}\right)_{k}^{4}}{\left(q^{d} ; q^{d}\right)_{k}^{4}} q^{(d-2 r) k} \\
& \quad \equiv q^{r(n+r-d n) / d} \frac{\left(q^{2 r} ; q^{d}\right)_{(d n-n-r) / d}}{\left(q^{d} ; q^{d}\right)_{(d n-n-r) / d}}[d n-n] \quad\left(\bmod \Phi_{n}(q)^{4}\right)
\end{aligned}
$$


By Lemma 2.2, the above $q$-congruence is true modulo $[n]$ and is therefore also true modulo $[n] \Phi_{n}(q)^{3}$. Further, if $d=2$ then by the condition in the theorem, one sees that $r<0$ and $-r<(n-r) / 2$ and so $\left(q^{2 r} ; q^{2}\right)_{(n-r) / 2}$ vanishes. This completes the proof.

Proof of Theorem 1.4. Let $d \geqslant 3$ and take $r=1$ and $M=n-1$ in (3.1). Noticing that $\left(q^{2} ; q^{d}\right)_{(d n-n-1) / d}$ is congruent to 0 modulo $\Phi_{n}(q)$ (we have mentioned this before) and $\left(q^{d} ; q^{d}\right)_{(d n-n-1) / d}$ is coprime with $\Phi_{n}(q)$, we see that (1.10) holds modulo $\Phi_{n}(q)^{2}$. By Lemma 2.2, it also holds modulo $[n]$. This proves the theorem.

\section{Concluding Remarks}

Using Lemma 2.1, we may also prove the following result similar to Lemma 2.2 .

Lemma 4.1. Let $d, n$ be positive integers with $\operatorname{gcd}(d, n)=1$. Let $r$ be an integer. Then

$$
\begin{aligned}
& \sum_{k=0}^{m}[2 d k+r] \frac{\left(q^{r} ; q^{d}\right)_{k}^{2 d}}{\left(q^{d} ; q^{d}\right)_{k}^{2 d}} q^{d(d-1-r) k} \equiv 0 \quad(\bmod [n]), \\
& \sum_{k=0}^{n-1}[2 d k+r] \frac{\left(q^{r} ; q^{d}\right)_{k}^{2 d}}{\left(q^{d} ; q^{d}\right)_{k}^{2 d}} q^{d(d-1-r) k} \equiv 0 \quad(\bmod [n]),
\end{aligned}
$$

where $0 \leqslant m \leqslant n-1$ and $d m \equiv-r(\bmod n)$.

Thus, the $q$-congruence (1.9) (i.e., [10, Theorem 1]) can be further strengthened as follows.

Theorem 4.2. Let $d, n, r$ be integers satisfying $d \geqslant 3, r \leqslant d-2$, and $n \geqslant d-r$, such that $d$ and $r$ are coprime, and $n \equiv-r(\bmod d)$. Then

$$
\sum_{k=0}^{M}[2 d k+r] \frac{\left(q^{r} ; q^{d}\right)_{k}^{2 d}}{\left(q^{d} ; q^{d}\right)_{k}^{2 d}} q^{d(d-1-r) k} \equiv 0 \quad\left(\bmod [n] \Phi_{n}(q)^{3}\right),
$$

where $M=(d n-n-r) / d$ or $n-1$.

Similarly to the proof of Lemma 2.3, we can also prove the following generalization of [13, Theorem 4.2].

Theorem 4.3. Let $d$ and $n$ be positive integers with $n \equiv 1(\bmod d)$. Then, modulo $[n]\left(1-a q^{n}\right)\left(a-q^{n}\right)$,

$$
\begin{aligned}
& \sum_{k=0}^{m}[2 d k+1] \frac{\left(a q, q / a, q / b, q ; q^{d}\right)_{k}}{\left(a q^{d}, q^{d} / a, b q^{d}, q^{d} ; q^{d}\right)_{k}} b^{k} q^{(d-2) k} \\
& \equiv \frac{(b / q)^{(n-1) / d}\left(q^{2} / b ; q^{d}\right)_{(n-1) / d}}{\left(b q^{d} ; q^{d}\right)_{(n-1) / d}}[n],
\end{aligned}
$$

where $m=(n-1) / d$ or $n-1$. 
Moreover, using the Chinese remainder theorem for coprime polynomials, we may prove that, for such $n$ and $d$, modulo $[n]\left(1-a q^{n}\right)\left(a-q^{n}\right)\left(b-q^{n}\right)$,

$$
\begin{aligned}
\sum_{k=0}^{m}[2 d k+1] \frac{\left(a q, q / a, q / b, q ; q^{d}\right)_{k}}{\left(a q^{d}, q^{d} / a, b q^{d}, q^{d} ; q^{d}\right)_{k}} b^{k} q^{(d-2) k} \\
\equiv \\
\quad \frac{(b / q)^{(n-1) / d}\left(q^{2} / b ; q^{d}\right)_{(n-1) / d}}{\left(b q^{d} ; q^{d}\right)_{(n-1) / d}} \frac{\left(b-q^{n}\right)\left(a b-1-a^{2}+a q^{n}\right)}{(a-b)(1-a b)}[n] \\
\quad+\frac{\left(q, q^{d-1} ; q^{d}\right)_{(n-1) / d}}{\left(a q^{d}, q^{d} / a ; q^{d}\right)_{(n-1) / d}} \frac{\left(1-a q^{n}\right)\left(a-q^{n}\right)}{(a-b)(1-a b)}[n] .
\end{aligned}
$$

For $d=1$, the $q$-congruence (4.2) is just an identity (with a telescoping truncated sum). For $d=2$, letting $b=1$ in (4.3), we get (1.6), which further reduces to (1.3) when $m=(n-1) / 2$ and $a$ tends to 1 . This is what the first author obtained in [6]. For $d \geqslant 3$, we are unable to deduce, by first letting $b=1$ and then taking $a \rightarrow 1$, a concrete interesting $q$-congruence modulo $\Phi_{n}(q)^{4}$ from (4.3) (because there appears to be no simple formula for the limit).

We conclude our paper with the following two conjectural $q$-supercongruences related to Theorem 3.1. (In the following conjectures, we actually consider the difference of the sums in (3.1), determined by the two endpoints $M=n-1$, and $M=(d n-n-r) / d$. We observed that this difference has a factor, as specified in Conjectures 4.4 and 4.5.) In the case $d=2$ and $r=1$ they coincide and were recently confirmed by the first author $[6$, Theorem 6.1].

Conjecture 4.4. Let $d, n$ be positive integers and $r$ a (possibly negative) integer with $d n-n \geqslant r \geqslant d-n$, such that $d$ and $r$ are coprime, and $n \equiv-r(\bmod d)$. Then, modulo $[n] \Phi_{n}(q)\left(1-a q^{d n-n}\right)\left(a-q^{d n-n}\right)\left(b-q^{d n-n}\right)$,

$$
\sum_{k=(d n-n-r+d) / d}^{n-1}[2 d k+r] \frac{\left(a q^{r}, q^{r} / a, q^{r} / b, q^{r} ; q^{d}\right)_{k}}{\left(a q^{d}, q^{d} / a, b q^{d}, q^{d} ; q^{d}\right)_{k}} b^{k} q^{(d-2 r) k} \equiv 0 .
$$

Conjecture 4.5. Let $d, n$ be positive integers and $r$ a (possibly negative) integer with $n \geqslant r \geqslant n-d n+d$, such that $d$ and $r$ are coprime, and $n \equiv r(\bmod d)$. Then, modulo $[n] \Phi_{n}(q)\left(1-a q^{n}\right)\left(a-q^{n}\right)\left(b-q^{n}\right)$,

$$
\sum_{k=(n-r+d) / d}^{n-1}[2 d k+r] \frac{\left(a q^{r}, q^{r} / a, q^{r} / b, q^{r} ; q^{d}\right)_{k}}{\left(a q^{d}, q^{d} / a, b q^{d}, q^{d} ; q^{d}\right)_{k}} b^{k} q^{(d-2 r) k} \equiv 0 .
$$

Funding Open access funding provided by Austrian Science Fund (FWF).

Open Access. This article is licensed under a Creative Commons Attribution 4.0 International License, which permits use, sharing, adaptation, distribution and reproduction in any medium or format, as long as you give appropriate credit to the original author(s) and the source, provide a link to the Creative Commons licence, 
and indicate if changes were made. The images or other third party material in this article are included in the article's Creative Commons licence, unless indicated otherwise in a credit line to the material. If material is not included in the article's Creative Commons licence and your intended use is not permitted by statutory regulation or exceeds the permitted use, you will need to obtain permission directly from the copyright holder. To view a copy of this licence, visit http://creativecommons. org/licenses/by/4.0/.

\section{References}

[1] Berndt, B.C.: Ramanujan's Notebooks, Part IV. Springer, New York (1994)

[2] Gasper, G., Rahman, M.: Encyclopedia of Mathematics and Its Applications. Basic Hypergeometric Series, vol. 96, 2nd edn. Cambridge University Press, Cambridge (2004)

[3] Gorodetsky, O.: q-Congruences, with applications to supercongruences and the cyclic sieving phenomenon. Int. J. Number Theory 15, 1919-1968 (2019)

[4] Guo, V.J.W.: A q-analogue of the (A.2) supercongruence of Van Hamme for primes $p \equiv 1(\bmod 4)$. Rev. R. Acad. Cienc. Exactas Fís. Nat. Ser. A Mat. 114, $123(2020)$

[5] Guo, V.J.W.: q-Analogues of Dwork-type supercongruences. J. Math. Anal. Appl. 487, 124022 (2020)

[6] Guo, V.J.W.: q-Supercongruences modulo the fourth power of a cyclotomic polynomial via creative microscoping. Adv. Appl. Math. 120, 102078 (2020)

[7] Guo, V.J.W.: A further $q$-analogue of Van Hamme's (H.2) supercongruence for primes $p \equiv 3(\bmod 4)$. Int. J. Number Theory (to appear)

[8] Guo, V.J.W., Schlosser, M.J.: Proof of a basic hypergeometric supercongruence modulo the fifth power of a cyclotomic polynomial. J. Differ. Equ. Appl. 25, 921-929 (2019)

[9] Guo, V.J.W., Schlosser, M.J.: Some new $q$-congruences for truncated basic hypergeometric series: even powers. Results Math. 75, 1 (2020)

[10] Guo, V.J.W., Schlosser, M.J.: A family of $q$-hypergeometric congruences modulo the fourth power of a cyclotomic polynomial. Israel J. Math. (to appear)

[11] Guo, V.J.W., Schlosser, M.J.: Some $q$-supercongruences from transformation formulas for basic hypergeometric series. Constr. Approx. (to appear)

[12] Guo, V.J.W., Wang, S.-D.: Some congruences involving fourth powers of central q-binomial coefficients. Proc. R. Soc. Edinb. Sect. A 150, 1127-1138 (2020)

[13] Guo, V.J.W., Zudilin, W.: A q-microscope for supercongruences. Adv. Math. 346, 329-358 (2019)

[14] Guo, V.J.W., Zudilin, W.: A common $q$-analogue of two supercongruences. Results Math. 75, 46 (2020)

[15] Liu, J.-C.: On a congruence involving $q$-Catalan numbers. C. R. Math. Acad. Sci. Paris 358, 211-215 (2020)

[16] Liu, J.-C., Petrov, F.: Congruences on sums of $q$-binomial coefficients. Adv. Appl. Math. 116, 102003 (2020) 
[17] Long, L.: Hypergeometric evaluation identities and supercongruences. Pac. J. Math. 249, 405-418 (2011)

[18] Ni, H.-X., Pan, H.: Some symmetric $q$-congruences modulo the square of a cyclotomic polynomial. J. Math. Anal. Appl. 481, 123372 (2020)

[19] Ramanujan, S.: Modular equations and approximations to $\pi$. Quart. J. Math. Oxford Ser. (2) 45, 350-372 (1914)

[20] Sun, Z.-W.: Super congruences and Euler numbers. Sci. China Math. 54, 25092535 (2011)

[21] Straub, A.: Supercongruences for polynomial analogs of the Apéry numbers. Proc. Am. Math. Soc. 147, 1023-1036 (2019)

[22] Tauraso, R.: Some $q$-analogs of congruences for central binomial sums. Colloq. Math. 133, 133-143 (2013)

[23] Van Hamme, L.: Some conjectures concerning partial sums of generalized hypergeometric series. In: p-Adic Functional Analysis (Nijmegen, 1996). Lecture Notes in Pure and Applied Mathematics, vol. 192, pp. 223-236. Dekker, New York (1997)

[24] Wang, X., Yue, M.: A $q$-analogue of the (A.2) supercongruence of Van Hamme for any prime $p \equiv 3(\bmod 4)$. Int. J. Number Theory 16, 1325-1335 (2020)

[25] Wang, X., Yue, M.: Some $q$-supercongruences from Watson's $8 \phi_{7}$ transformation formula. Results Math. 75, 71 (2020)

[26] Zudilin, W.: Ramanujan-type supercongruences. J. Number Theory 129, 18481857 (2009)

[27] Zudilin, W.: Congruences for $q$-binomial coefficients. Ann. Combin. 23, 11231135 (2019)

Victor J. W. Guo

School of Mathematics and Statistics

Huaiyin Normal University

Huai'an 223300 Jiangsu

People's Republic of China

e-mail: jwguo@hytc.edu.cn

Michael J. Schlosser

Fakultät für Mathematik

Universität Wien

Oskar-Morgenstern-Platz 1

1090 Vienna

Austria

e-mail: michael.schlosser@univie.ac.at

Received: July 24, 2020.

Accepted: September 11, 2020.

Publisher's Note Springer Nature remains neutral with regard to jurisdictional claims in published maps and institutional affiliations. 